\title{
Characterization of dissolved organic matter using high-performance liquid chromatography (HPLC)-size exclusion chromatography (SEC) with a multiple wavelength absorbance detector
}

\author{
Mingquan Yan ${ }^{\mathrm{a}, \mathrm{b}, *}$, Gregory Korshin ${ }^{\mathrm{b}}$, Dongsheng Wang ${ }^{\mathrm{c}}$, Zhenxiao Cai $^{\mathrm{b}}$ \\ ${ }^{a}$ Department of Environmental Engineering, Peking University, The Key Laboratory of Water and Sediment Sciences, Ministry of Education, Beijing 100871, China \\ ${ }^{\mathrm{b}}$ Department of Civil and Environmental Engineering, University of Washington, Box 352700, Seattle, WA 98195-2700, United States \\ ' State Key Laboratory of Environmental Aquatic Chemistry, Research Center for Eco-Environmental Sciences, CAS, POB 2871, Beijing 100085, China
}

\section{A R T I C L E I N F O}

\section{Article history:}

Received 21 October 2011

Received in revised form 18 January 2012

Accepted 18 January 2012

Available online 25 February 2012

\section{Keywords:}

HPLC-SEC

Multiple wavelength absorbance detector

Molecular weight

DOM

\begin{abstract}
A B S T R A C T
High-performance liquid chromatography-size exclusion chromatography (HPLC-SEC) coupled with a multiple wavelength absorbance detector (200-445 nm) was used in this study to investigate the apparent molecular weight (AMW) distributions of dissolved organic matter (DOM). Standard DOM, namely humic acid, fulvic acid and hydrophilic acid, from the Suwannee River were tested to ascertain the performance and sensitivity of the method. In addition to four compounds groups: humic substances (Peak 1, AMW 16 kD), fulvic acids (Peak 2, AMW $11 \mathrm{kD}$ ), low AMW acids (Peak 3, AMW 5 kD), and low AMW neutral and amphiphilic molecules, proteins and their amino acid building blocks (Peak 4, AMW $3 \mathrm{kD}$ ), an new group that appears to include low AMW, 6-10 kD, humic substances was found based on investigating the spectra at various elution times. The spectroscopic parameter $S_{>365}$ (slope at wavelengths $>365 \mathrm{~nm}$ ) was determined to be a good predictor of the AMW of the DOM. The detector wavelength played an important role in evaluating the AMW distribution. For some fractions, such as the humic and low AMW non-aromatic substances, the error in measurement was $\pm 30 \%$ as determined by twodimensional chromatograms detected at an artificially selected wavelength. HPLC-SEC with multiple wavelength absorbance detection was found to be a useful technique for DOM characterization. It characterized the AMW distributions of DOM more accurately and provided additional, potentially important information concerning the properties of DOM with varying AMWs.
\end{abstract}

(c) 2012 Elsevier Ltd. All rights reserved.

\section{Introduction}

Dissolved organic matter (DOM) is ubiquitous in natural waters and is derived from plant or terrestrial (allochtonous) and algal (autochthonous) sources. It is composed of relatively high apparent molecular weight (AMW) humic and fulvic acids, as well as low AMW proteins, organic acids, carbohydrates and other compounds (Leenheer and Croue, 2003). The molecular properties of DOM, notably its molecular weight, strongly affect its reactivity in both natural and engineered aquatic systems (Amy et al., 1987; Her et al., 2002a; Leenheer and Croue, 2003; Yan et al., 2006, 2007; Korshin et al., 2009). High-performance liquid chromatographysize exclusion chromatography (HPLC-SEC) is one of the most widely used techniques to characterize DOM due to its important advantages, such as little need for DOM pre-concentration, small

\footnotetext{
* Corresponding author at: Department of Environmental Engineering, Peking University, The Key Laboratory of Water and Sediment Sciences, Ministry of Education, Beijing 100871, China. Tel.: +86 10 62755914-81; fax: +86 1062756526 .

E-mail address: yanmq@pku.edu.cn (M. Yan).
}

injection volume, and ease and speed of analysis (Chin et al., 1994; Her, 2003; Korshin et al., 2009). In principle, the separation of molecules in a SEC column is based on size, and compounds with lower AMW are eluted later than those with higher AMW. However, the interpretation of HPLC-SEC data is greatly affected by the type of post-column detection used (Her, 2003; Korshin et al., 2009), typically UV absorbance, fluorescence, or multi-angle light scattering (MALS) (Baker, 2001; Wong et al., 2002; Her, 2003; Allpike et al., 2005; Akagi et al., 2007; Liu et al., 2010a). Post-column absorbance detection is most common due to its sensitivity and relatively low cost (O'Loughlin and Chin, 2001; Her et al., 2002b, 2008; Hur and Kim, 2009). DOM chromophores produce overlapping bands, so their absorbance spectra are largely featureless and cannot be deconvoluted to determine the contributions of discrete functional groups (Korshin et al., 1997, 2009; Her et al., 2008). In most cases, such measurements have been limited to using a single wavelength (e.g., $254 \mathrm{~nm}$ ).

However, a number of phenomenological parameters (e.g., $\mathrm{SUVA}_{254}$, ratios of DOM absorbances at fixed wavelengths, and slopes of DOM absorbance vs. wavelength) have been correlated 
with important DOM properties (e.g., aromaticity and apparent molecular weight) that have been estimated using structure-sensitive methods (e.g., ${ }^{13} \mathrm{C}$ NMR).

These phenomenological parameters have rarely been used to interpret HPLC-SEC data. For instance, Her et al. (2008) used the ratio of absorbance of varying-MW fractions measured at $210 \mathrm{~nm}$ and $254 \mathrm{~nm}$ to estimate the relative contributions of fulvic acids and microbial biopolymers. Korshin et al. (2009) used the ratio of the absorbance slopes of wavelengths $254-272 \mathrm{~nm}$ vs. 220$230 \mathrm{~nm}$ (ISA) to evaluate the DOM removal and DBP formation potential.

In this paper, we examined the performance of alternative absorbance-based indexes (e.g., absorption ratios and slopes). Our conclusions were based on the analysis of absorbance spectra, from $200 \mathrm{~nm}$ to $445 \mathrm{~nm}$, of HPLC-SEC fractions with varying AMWs. This approach was applied to the Suwannee River humic acid, fulvic acid and hydrophilic acid standards to determine the performance and sensitivity of the method.

\section{Methods and materials}

\subsection{Samples}

Suwannee River humic acid (SRHA) (1R101H) and Suwannee River fulvic acid (SRFA) (1S101F) standards were obtained from the International Humic Substances Society (IHSS). Suwannee River hydrophilic acid (SRHPiA) was isolated and provided for this study by Dr. J.A. Leenheer. SRHPiA was obtained by passing an acidified ( $\mathrm{pH} 2$ ) sample of Suwannee River organic matter, eluted from column containing Amberlite XAD-8 resin, through a column containing Amberlite XAD-4 resin. This sample was then eluted from the XAD-4 resin with sodium hydroxide. SRHA, SRFA and SRHPiA solutions were prepared at a $5.0 \mathrm{mg} \mathrm{L}^{-1}$ final TOC concentration in Milli-Q water. Dissolved organic carbon (DOC) was analyzed using a Shimadzu TOC-Vcsh carbon analyzer. The ionic strength of each was maintained at $0.04 \mathrm{M}$ by adding the requisite amount of $\mathrm{NaClO}_{4}$.

\subsection{HPLC-SEC analyses}

The HPLC-SEC analysis used a DIONEX Ultimate 3000 HPLC system. Isocratic flow of $0.01 \mathrm{M} \mathrm{NH}_{4} \mathrm{HCO}_{3}$ was delivered through an Agilent PL aquagel-OH 30 column $(300 \times 7.5,8 \mu \mathrm{m})$ at $0.5 \mathrm{~mL} \mathrm{~min}^{-1}$. Elution profiles were monitored with an Ultimate 3000 diode array detector, which acquired absorbance spectra from $200 \mathrm{~nm}$ to $445 \mathrm{~nm}$ at a resolution of $1.0 \mathrm{~nm}$.

A highly linear calibration curve for the apparent molecular weight (AMW) was generated using polyethylene glycol (PEG) standards (Agilent EasiVial PEG) (see Fig. 1S in Supplementary information (SI)), but it suggested that the AMW of the DOM was much larger than that reported by other researchers (Chin et al., 1994). Therefore, although the HPLC-SEC profiles are useful for comparisons among samples in a particular experiment, the absolute AMW values should not be interpreted literally (Cai and Benjamin, 2011).

The absorbance spectra of DOM samples were recorded in a $5 \mathrm{~cm}$ quartz cell on a Perkin-Elmer Lambda $18 \mathrm{UV} /$ vis spectrophotometer using wavelengths from $200 \mathrm{~nm}$ to $600 \mathrm{~nm}$.

\subsection{AMW evaluation}

AMW distributions can be represented by number-averaged AMW $\left(M_{n}\right)$, weight-averaged MW $\left(M_{w}\right)$, and polydispersity ( $p$, $\left.M_{w} / M_{n}\right) . M_{n}$ and $M_{w}$ values were determined using the following Eqs. (1) and (2):
$M_{w}=\frac{\sum_{i=1}^{n}\left(b_{i} \mathrm{MW}_{i}\right)}{\sum_{i=1}^{n} b_{i}}$

$M_{n}=\frac{\sum_{i=1}^{n} b_{i}}{\sum_{i=1}^{n}\left(b_{i} / \mathrm{MW}_{i}\right)}$

$p=\frac{M_{w}}{M_{n}}$

where $b_{i}$ and $\mathrm{MW}_{i}$ are the height of the chromatogram and the MW of an organic matter sample corresponding to retention time $i$, respectively (Chin et al., 1994).

\section{Theoretical aspects of the interpretation of absorbance spectra}

The energies of various types of molecular orbits differ significantly. It was reported that most detectable UV absorbance by organic compounds is based on transitions of $n$ or $\pi$ electrons to the $\pi^{*}$ excited state. The energies required for these processes bring the absorption peaks into an experimentally convenient spectral region (200-700 $\mathrm{nm}$ ) (Her et al., 2002b). The absorbance spectra of pure, model aromatic compounds can often be represented by three bands in the UV region from $\pi \rightarrow \pi^{*}$ transitions: the electron-transfer (ET) band centered at $254 \mathrm{~nm}$, the benzenoid (Bz) band centered at $203 \mathrm{~nm}$, and the local excitation (LE) band centered at $180 \mathrm{~nm}$. The ratio of the intensities of the electron transfer and benzenoid bands $\left(A_{\mathrm{ET}} / A_{\mathrm{Bz}}\right.$, or $\left.A_{254} / A_{203}\right)$ is quite sensitive to substitutions on the rings, increasing dramatically when the rings are substituted with activating functional groups (e.g., hydroxyl, ester, and carboxyl) (Korshin et al., 1997; Li et al., 2006). The main DOM functional groups associated with non-aromatic groups display absorption maxima only at shorter wavelengths, and, in the case of the non-conjugated form, the absorption maxima wavelengths were $206 \mathrm{~nm}$ (for carboxylic acid and esters) and $210 \mathrm{~nm}$ (for amides) in water for the $n \rightarrow \pi^{*}$ transition. When carboxylic acid or esters are conjugated, the peak maxima absorption occurs by $\pi \rightarrow \pi^{*}$ transitions at an experimentally detectable spectral region (longer than $200 \mathrm{~nm}$ ), and due to a substituent effects, the peak maxima absorption shifts to longer wavelengths from the base absorption of $187 \mathrm{~nm}$ in water. The absorption band increase is dependent upon the substituent type and its position on the unsaturated carbonyl chromospheres (Her et al., 2008). The overall spectrum of a DOM sample represents a superposition of these bands. However, by refining spectral parameters, some useful information could be obtained from the overall spectrum of a DOM sample. A review and relevant values of SRHA, SRFA and SRHPiA are provided in Table 1.

The conventional absorbance spectra of SRHA, SRFA and SRHPiA are shown in Fig. 1a. Except for a shoulder located in the 250$270 \mathrm{~nm}$ range of wavelengths, these spectra did not exhibit any prominent peaks. In all cases, the absorbance gradually decreased at increased wavelengths. While the TOC concentrations of these three samples were equal, the absorbance intensities were different. Due to its higher aromaticity, SRHA absorbance values were twice as high as those of SRFA and SRHPiA. When the spectra were normalized by their absorbance measured at an a priori selected reference wavelength (e.g., $220 \mathrm{~nm}$ ), additional properties of these spectra became more evident, as shown in Fig. 1b. At wavelengths $>250 \mathrm{~nm}$, the absorbance of SRHA was higher than that of SRFA and SRHPiA. At the same time, the normalized spectrum of SRHPiA was slightly higher than that of SRFA, and close to that of SRHA, in the 250-270 nm range.

Several indexes were derived based on the ratios of the absorbance of SRHA, SRFA and SRHPiA at varying wavelengths and are presented in Table 1 . It was also recognized that the 

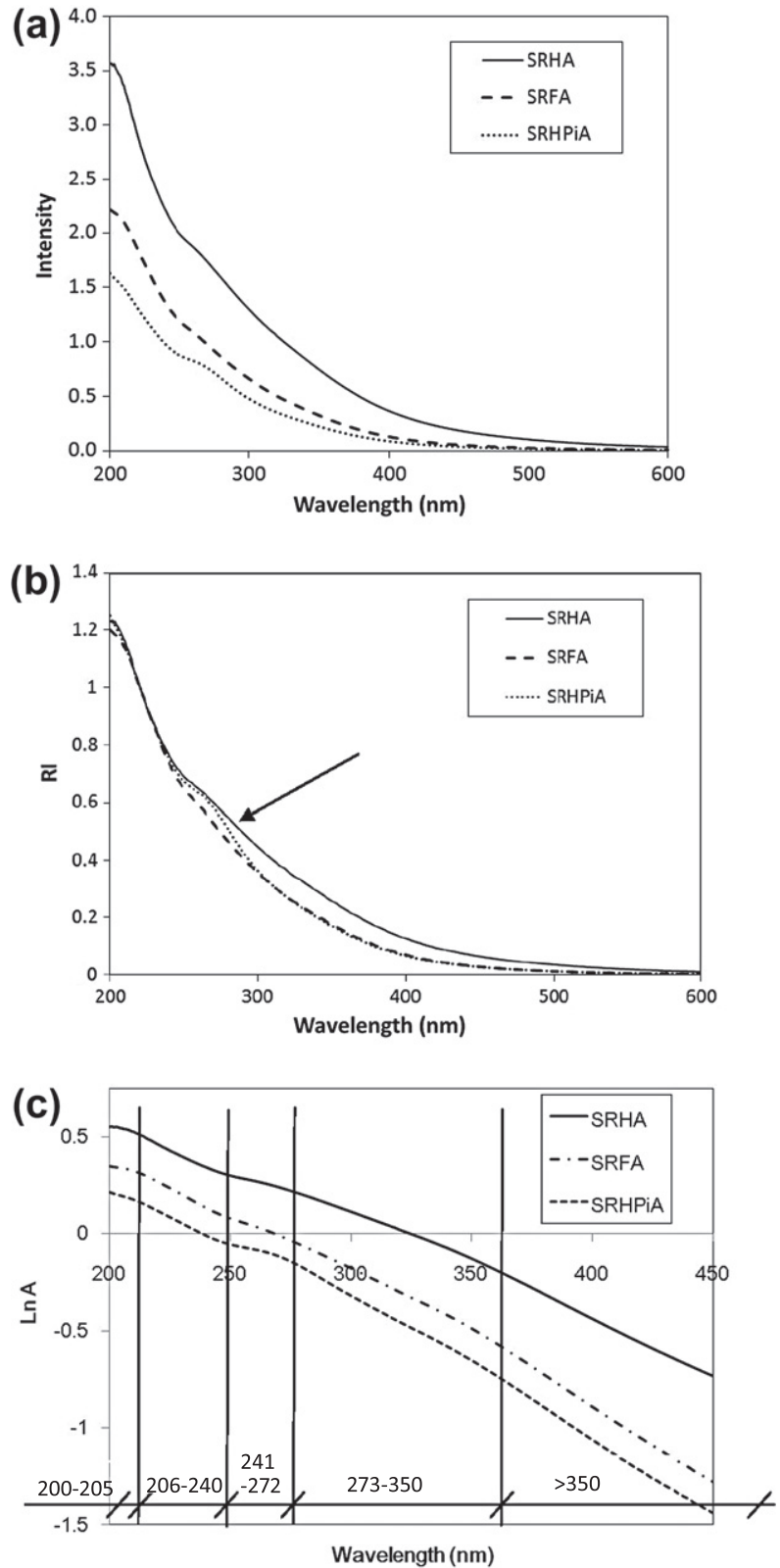

Fig. 1. Absorbance spectra of SRHA, SRFA and SRHPiA scanned by UV spectrophotometer. (a) Absorbance spectra; (b) absorbance spectra normalized to the reference absorbance at $220 \mathrm{~nm}$ and (c) natural log-transformed absorption spectra.

corresponding to P2 was not obvious because SRFA had a higher $A_{210} / A_{254}$ value. There were only two peaks found for SRHPiA at P2 and P4. The values of $A_{210} / A_{254}$ showed similar values at two AMW ranges, $14-20 \mathrm{kD}$ and $6-10 \mathrm{kD}$.

The $A_{280} / A_{350}$ values for the three samples exhibited similar trends when AMW decreased. These ratios decreased gradually at AMWs larger than $6 \mathrm{kD}$, except for a peak at the AMWs of 11$14 \mathrm{kD}$. This may indicate that DOM with an AMW of $11-14 \mathrm{kD}$ had specific groups with non-aromatic functional character. The $A_{280} / A_{350}$ values increased at an AMW of $6 \mathrm{kD}$ and decreased gradually as the AMW decreased.

The behavior of the spectral slopes calculated for wavelength ranges $206-240 \mathrm{~nm}$ and $273-350 \mathrm{~nm}$ is shown in Fig. 4. These data showed similar trends to those observed for the $A_{210} / A_{254}$ and $A_{280} /$ $A_{350}$ values.

The results shown in Figs. 2-4 and data of prior research (Huber and Frimmel, 1994; O'Loughlin and Chin, 2001; Her et al., 2002a,b;
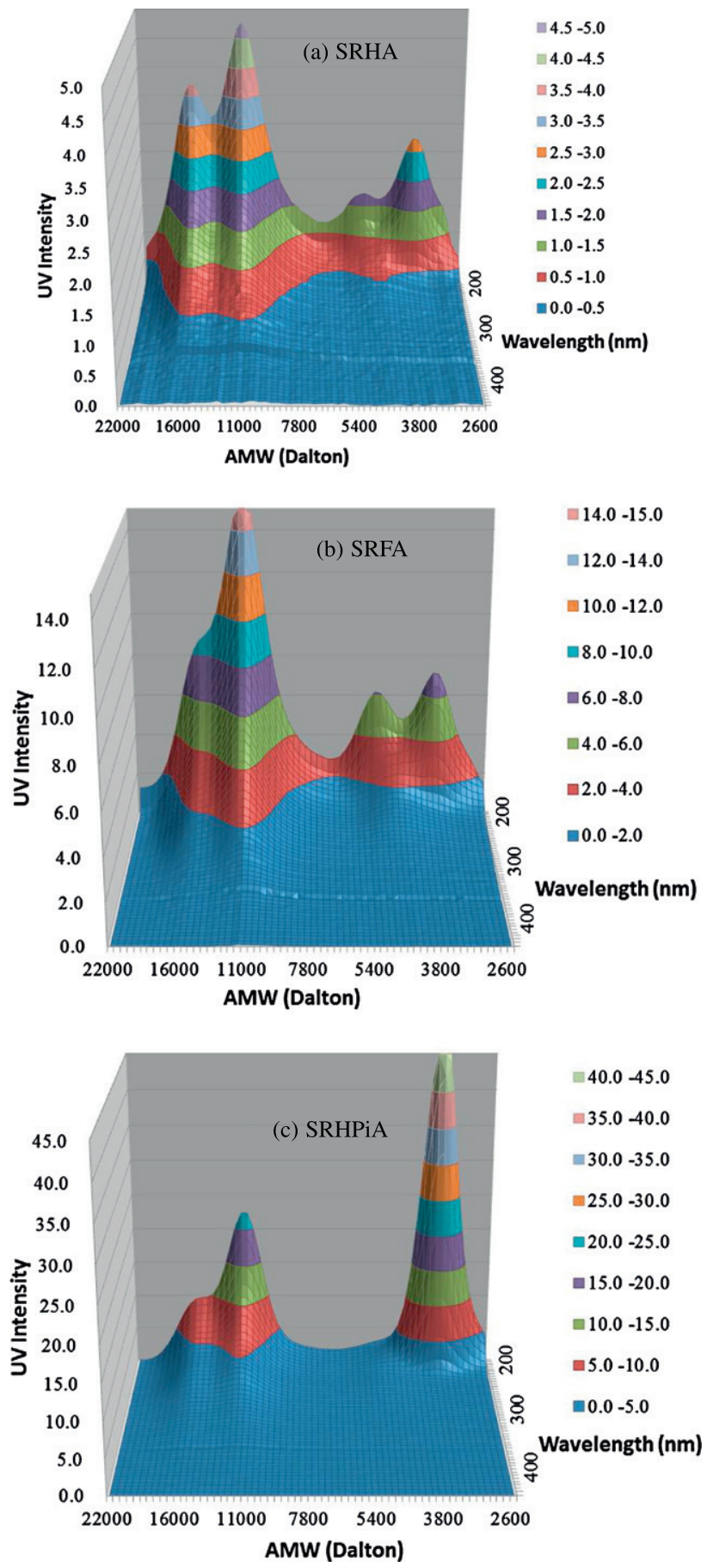

Fig. 2. HPLC-SEC chromatograms for (a) SRHA, (b) SRFA and (c) SRHPiA.

Korshin et al., 2009; Liu et al., 2010b; Huber et al., 2011) revealed that DOM can be divided into five distinct component fractions based on AMW. The first fraction (F1) had an AMW larger than $14 \mathrm{kD}$ (observed at elution times from $14.2 \mathrm{~min}$ to $14.7 \mathrm{~min}$ ), corresponded to P1 and was likely comprised of humic substances (Rosenberger et al., 2006) that had relatively high aromaticity and contributions from functional groups with conjugated bonds. This fraction had a relatively low $A_{210} / A_{254}$ and $S_{206-240}$ values and relatively high $A_{280} / A_{350}$ and $S_{273-350}$ values. The second fraction (F2) had an AMW of 10-14 kD (observed at elution times from $14.7 \mathrm{~min}$ to $15.2 \mathrm{~min}$ ). It corresponded to P2 and was comprised of fulvic acids, characterized by highly aromatic components and high conjugated no-aromatic activating functional groups. It had 

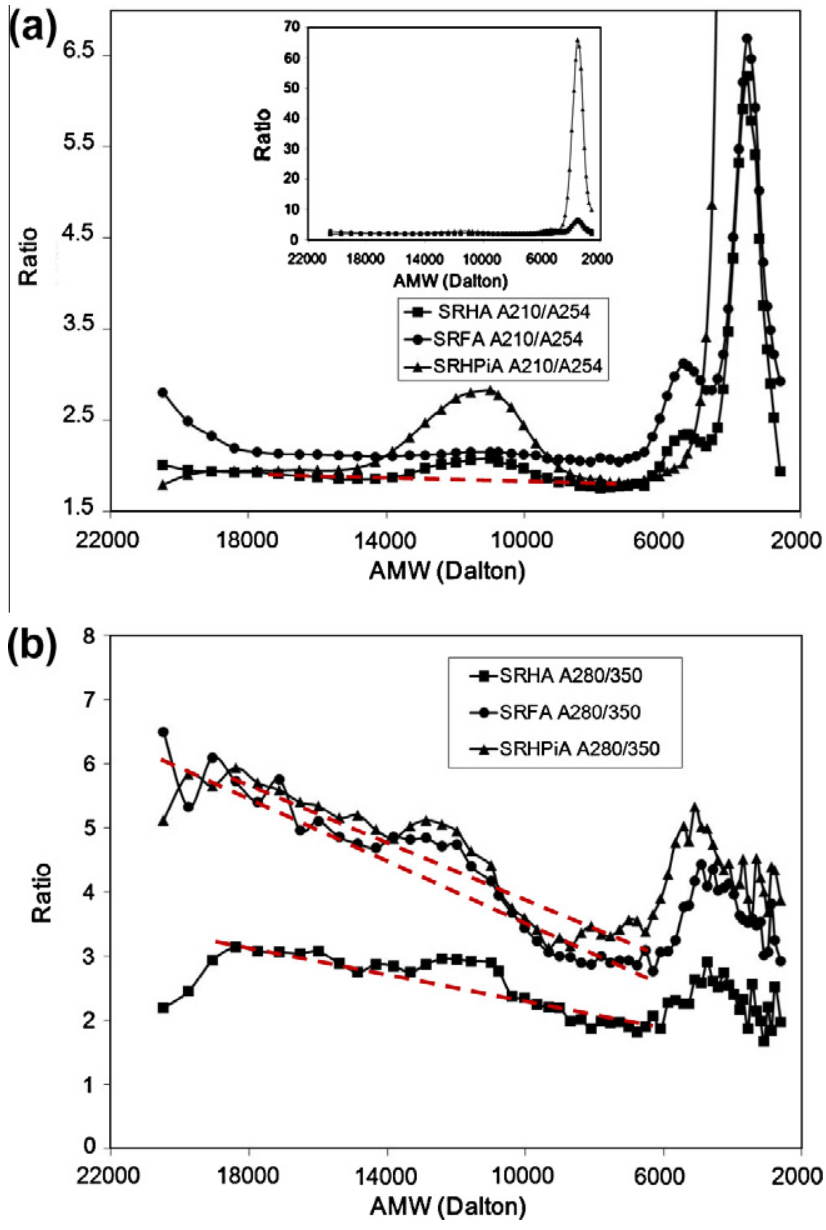

Fig. 3. Comparison of the absorption ratios of SRHA, SRFA and SRHPiA.

relatively higher $A_{210} / A_{254}, A_{280} / A_{350}, S_{206-240}$, and $S_{273-350}$ values. The third component (F3) had an AMW of $6-10 \mathrm{kD}$ (observed at elution times from $15.2 \mathrm{~min}$ to $15.8 \mathrm{~min}$ ). It corresponded to the tailing part of the second peak and appeared to include humic substances with relatively low AMWs and operationally defined building blocks (sub-units of humic substances), characterized by high aromatic components and low non-aromatic functional groups. It had relatively lower $A_{210} / A_{254}, A_{280} / A_{350}, S_{206-240}$, and $S_{273-350}$ values. The DOM with larger MWs contained more aliphatic carbons. The aliphatic $C$ chains had a lower content of $C=C$, carbon double bonds, and have higher UV absorptivity at shorter wavelengths. Conversely, the fractions with smaller MWs, such as aromatic structures, had a greater content of $\mathrm{C}=\mathrm{C}$ and have a higher UV absorptivity at longer wavelengths (Liu et al., 2010b). The fourth fraction (F4) had an AMW of 4-6 kD (observed at elution times of $15.8 \mathrm{~min}$ to $16.2 \mathrm{~min}$ ) and corresponded to $\mathrm{P} 3$ that has been generally assigned to low MW acids (O'Loughlin and Chin, 2001; Liu et al., 2010b). This fraction had relatively high $A_{210} / A_{254}, A_{280} /$ $A_{350}, S_{206-240}$, and $S_{273-350}$ values. The fifth fraction (F5) had an AMW lower than $4 \mathrm{kD}$ (observed at elution times of 16.2$17.0 \mathrm{~min}$ ) and corresponded to $\mathrm{P} 4$ that has been generally assigned to low MW neutrals and amphiphilics, proteins and their amino acid building blocks. This fraction had the highest $A_{210} / A_{254}, S_{206}$ 240 values and relatively low $A_{280} / A_{350}, S_{273-350}$ values.

\subsection{MW evaluation by UV spectra}

In previous studies, several parameters, such as $A_{365}\left|A_{250}, A_{465}\right|$ $A_{665}$, spectral slope in the $275-295 \mathrm{~nm}$ range and the ratio of slopes
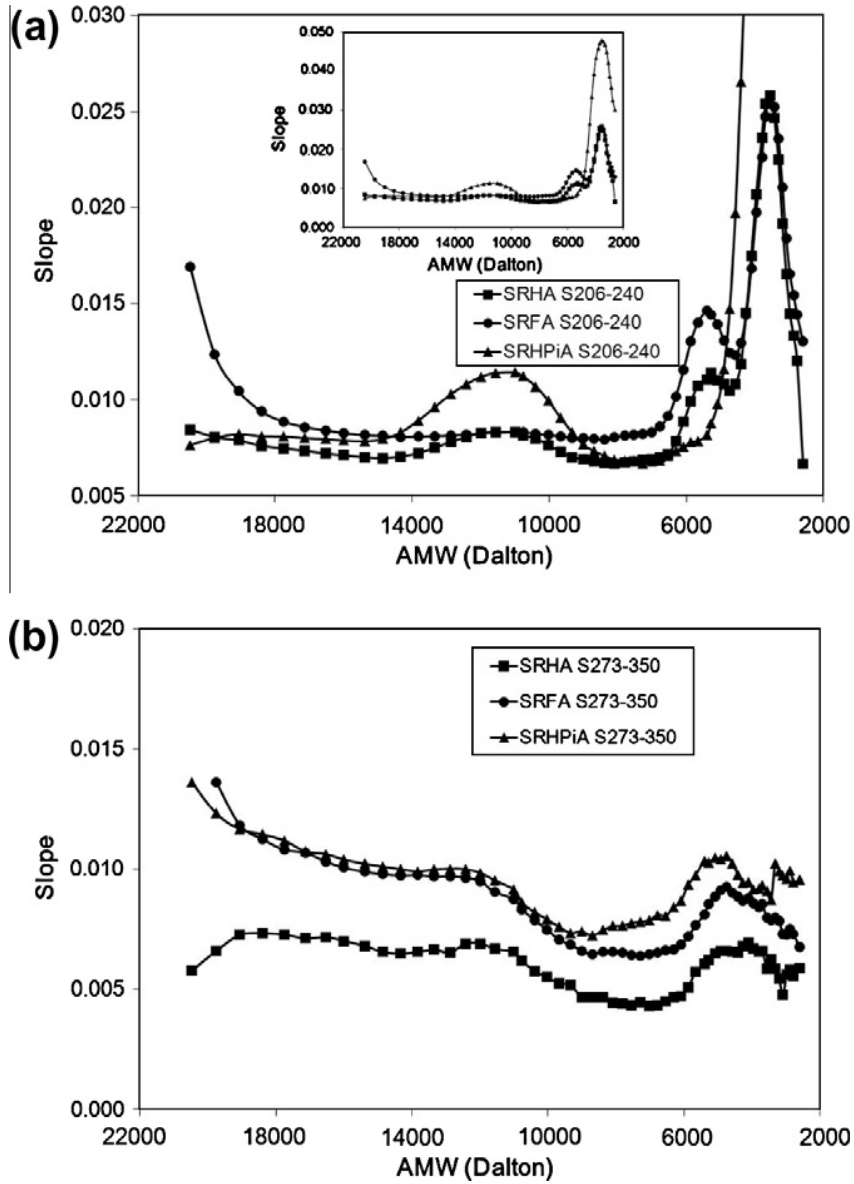

Fig. 4. Comparison of the slopes of absorbance spectra of SRHA, SRFA and SRHPiA.

of $275-295 \mathrm{~nm}$ and $350-450 \mathrm{~nm}$ ranges, were used to evaluate the relative sizes of DOM molecules found in fractions obtained from ultrafiltration or Gel filtration chromatography. In this paper, several spectroscopic parameters, including those outlined above, were used to evaluate the AMW base of the HPLC-SEC data at various elution times.

The $A_{365} / A_{254}$ values for SRHA, SRFA and SRHPiA are shown in Fig. 5a. In accord with the results of previous studies (Twardowski et al., 2004) the $A_{365} / A_{254}$ values were observed to generally increase with elution time. However, a notable peak located at elution times 15.2-15.8 min (with an AMW of $6-10 \mathrm{kD}$, as calculated by a standard AMW calibration curve) was observed for SRHA. It corresponded to F3, the fraction with low AMW humic substances and was characterized by highly aromatic components with high relative UV absorbance values at longer wavelengths. The MW of this DOM fraction was underestimated if $A_{365} / A_{254}$ was used to evaluate the AMW.

The $A_{365} / A_{254}$ values and the spectral slopes calculated for wavelengths $>365 \mathrm{~nm}\left(S_{>365}\right)$ represented a more accurate parameter with which to estimate the AMW of DOM molecules, as shown in Fig. 5b. The $S_{>365}$ values tended to decrease gradually and showed a more robust linear correlation vs. the elution time. However, the $S_{>365}$ values had higher scatter because the absorption intensities measured at longer wavelengths were closer to the detection limit of the detector.

\subsection{Error in MW distribution estimation}

The observation wavelength was evaluated to determine its effect on the estimate of AMW distributions in HPLC-SEC measurements. The HPLC-SEC spectra at various elution times were 
(a)
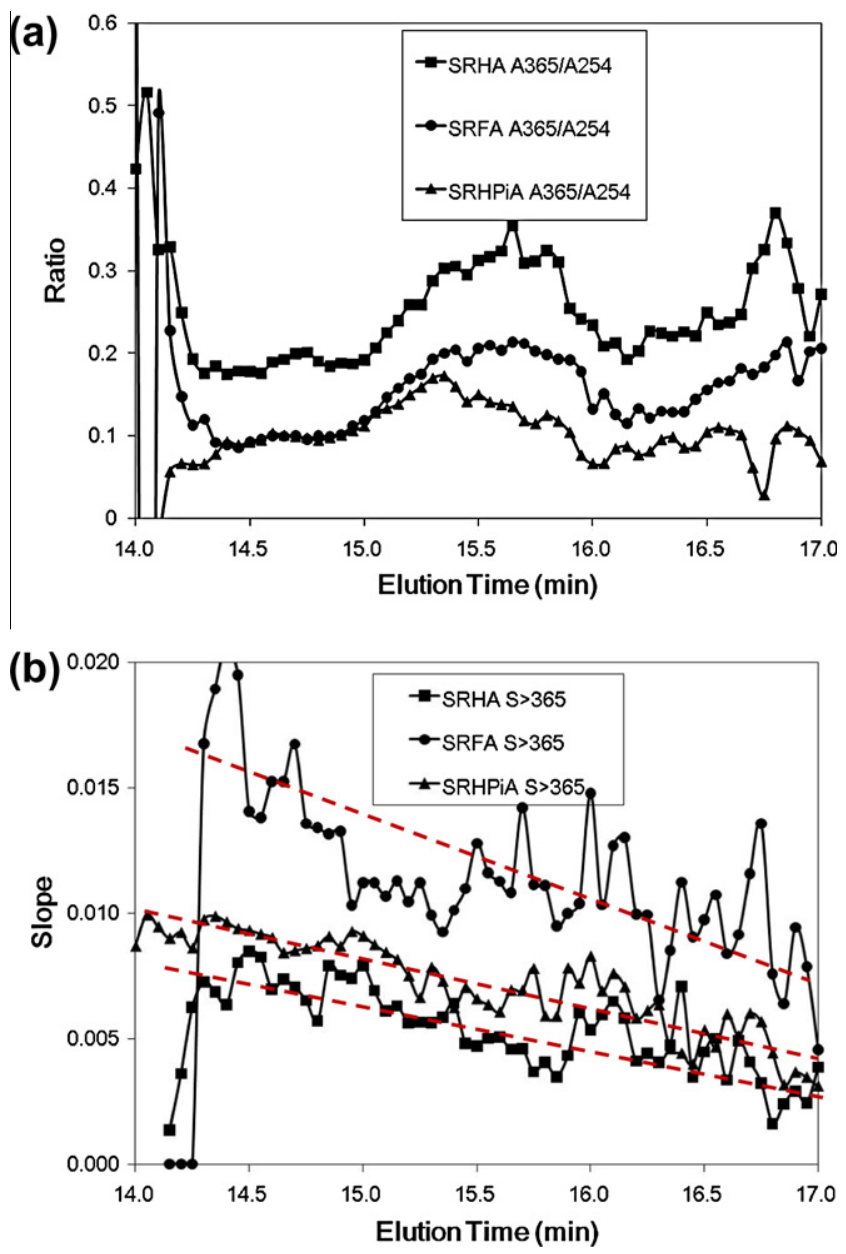

Fig. 5. MW estimation indexes of (a) $A_{356} / A_{254}$ and (b) $S_{>365}$.

normalized by the absorbance at a priori selected reference wavelength (e.g., $220 \mathrm{~nm}$ ). The data were processed further by subtracting the normalized absorbance spectrum of the DOM by absorbance intensity at the same reference wavelength. This calculation can be expressed in the following equation:

$R I_{w, t}=\frac{I_{w, t}}{I_{220, t}}-\frac{t_{w}}{t_{220}}$

where $R I_{w, t}$ is the error at wavelength $w$ and elution time $t(i=200-$ $445 \mathrm{~nm} ; w=14.2-17.0 \mathrm{~min}) . I_{w, t}$ is the intensity measured by HPLC-SEC at wavelength $w$ and elution time $t(w=200-445 \mathrm{~nm}$; $t=14.2-17.0 \mathrm{~min}) . I_{220, t}$ is the intensity measured by HPLC-SEC at wavelength $220 \mathrm{~nm}$ and elution time $t(t=14.2-17.0 \mathrm{~min}) . i_{w}$ is the intensity measured by UV/vis spectrophotometer at wavelength $w(w=200-445 \mathrm{~nm}) . i_{220}$ is the intensity measured by UV/vis spectrophotometer at wavelength $220 \mathrm{~nm}$.

The relative errors in MW distribution estimation for SRHA, SRFA and SRHPiA are shown in Fig. 6a-c, respectively. As shown in Fig. 6a for SRHA, there were two areas at wavelengths longer than $220 \mathrm{~nm}$ that have negative values beyond $10 \%$. These areas corresponded to DOM with an AMW larger than $10 \mathrm{kD}$ and smaller than $6 \mathrm{kD}$. Additionally, there were several peak areas found at wavelengths lower than $220 \mathrm{~nm}$, but these areas were overlooked in this study due to interference from inorganic ions at low wavelengths (e.g., $\mathrm{NO}_{3}^{-}, \mathrm{SO}_{4}^{2-}$, and $\mathrm{PO}_{4}^{3-}$ ) (Her et al., 2008; Korshin et al., 2009). SRFA and SRHPiA also showed the same phenomena as SRHA, though the areas and depths of the washes were different. The areas and depths of washes at AMWs larger than $10 \mathrm{kD}$ were smaller for SRFA and SRHPiA. Furthermore, the areas and depths
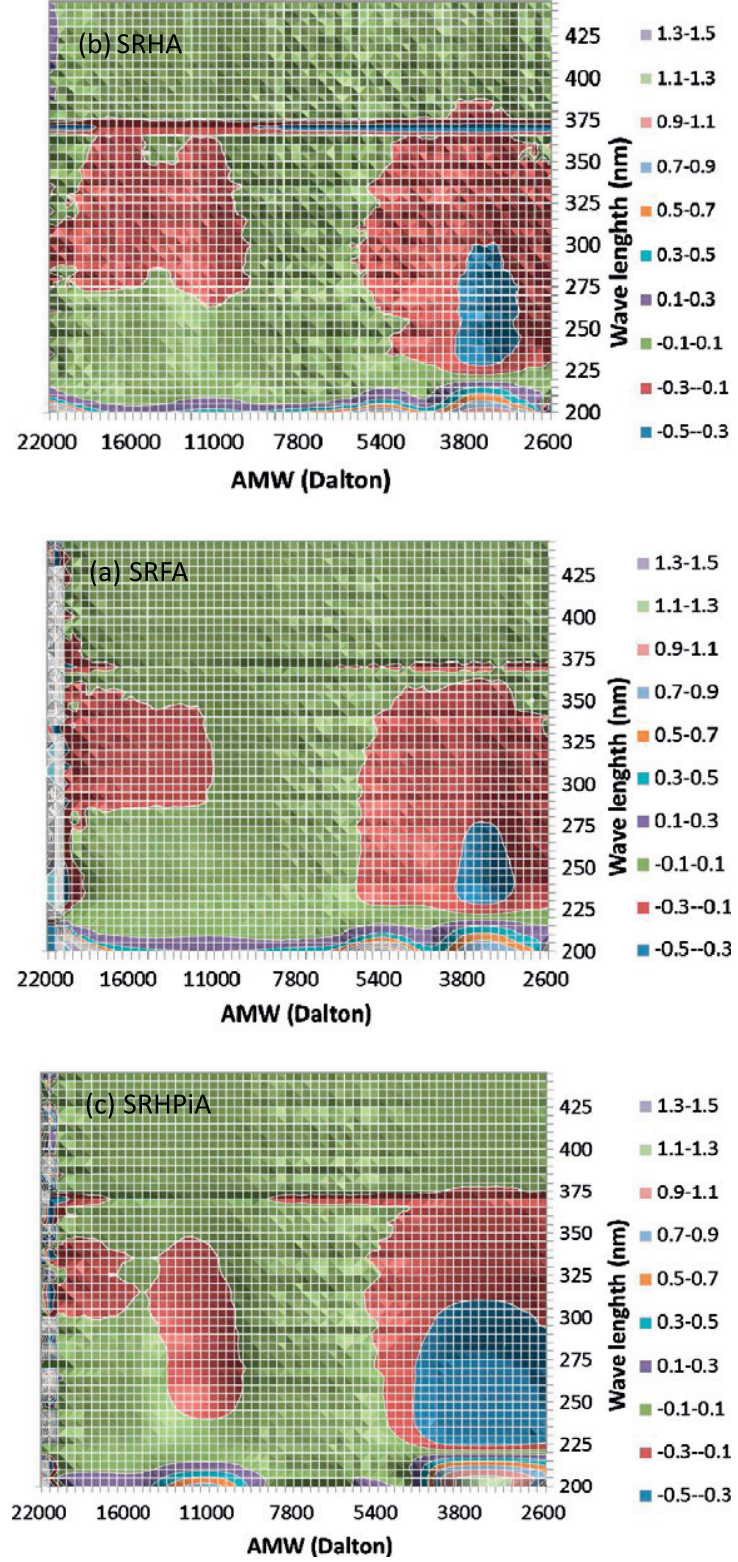

Fig. 6. Normalized HPLC-SEC chromatogram subtracted by the normalized absorbance spectra of (a) SRHA, (b) SRFA and (c) SRHPiA.

of the washes at AMWs smaller than $6 \mathrm{kD}$ are bigger for SRFA and especially SRHPiA.

There was an error in evaluating $M_{n}$ and $M_{w}$ based on Eqs. (1) and (2). The error changed with the wavelength utilized and the characteristics of the DOM. In general, the error was within $\pm 10 \%$. For some special fractions, such as humic substance or low WM non-aromatic matters, the error was greater than $30 \%$ at certain wavelengths.

The HPLC-SEC coupled with a multiple wavelength absorbance detector was a useful technique to characterize DOM. It not only characterized the MW distribution of the DOM more accurately, but it also provided some interesting information on DOM characteristics at various MWs. However, further work is needed to understand the UV spectra.

\section{Acknowledgements}

The authors are very grateful to Dr. Michael Dodd for their support for this research. This research was also founded by CNSF 
50808001, the China Postdoctoral Science Foundation and China Scholarship Council.

\section{Appendix A. Supplementary material}

HPLC-SEC calibration curve and the chromatograms for these samples measured at several representative wavelengths and are shown in Figs. 1S and $2 \mathrm{~S}$ in the Supplemental Information (SI), respectively. Supplementary data associated with this article can be found, in the online version, at doi:10.1016/j.chemosphere. 2012.01.029.

\section{References}

Akagi, J., Zsolnay, A., Bastida, F., 2007. Quantity and spectroscopic properties of soil dissolved organic matter (DOM) as a function of soil sample treatments: airdrying and pre-incubation. Chemosphere 69, 1040-1046.

Allpike, B.P., Heitz, A., Joll, C.A., Kagi, R.I., Abbt-Braun, G., Frimmel, F.H., Brinkmann, T., Her, N., Amy, G., 2005. Size exclusion chromatography to characterize DOC removal in drinking water treatment. Environ. Sci. Technol. 39, 2334-2342.

Amy, G.L., Collins, M.R., Kuo, C.J., King, P.H., 1987. Comparing gel-permeation chromatography and ultrafiltration for the molecular-weight characterization of aquatic organic-matter. J. Am. Water Works Assoc. 79, 43-49.

Baker, A., 2001. Fluorescence excitation-emission matrix characterization of some sewage-impacted rivers. Environ. Sci. Technol. 35, 948-953.

Cai, Z., Benjamin, M.M., 2011. NOM fractionation and fouling of low-pressure membranes in microgranular adsorptive filtration. Environ. Sci. Technol. 45 8935-8940.

Carder, K.L., Steward, R.G., Harvey, G.R., Ortner, P.B., 1989. Marine humic and fulvicacids - their effects on remote-sensing of ocean chlorophyll. Limnol. Oceanogr. $34,68-81$.

Chin, Y.P., Aiken, G., Oloughlin, E., 1994. Molecular-weight, polydispersity, and spectroscopic properties of aquatic humic substances. Environ. Sci. Technol. 28 1853-1858.

De Haan, H., De Boer, T., 1987. Applicability of light absorbance and fluorescence as measures of concentration and molecular size of dissolved organic carbon in humic Laken Tjeukemeer. Water Res. 21, 731-734.

Helms, J.R. Stubbins, A., Ritchie, J.D., Minor, E.C., Kieber, D.J., Mopper, K., 2008 Absorption spectral slopes and slope ratios as indicators of molecular weight, source, and photobleaching of chromophoric dissolved organic matter. Limnol. Oceanogr. 53, 955-969.

Her, N., 2003. Characterization of DOM as a function of MW by fluorescence EEM and HPLC-SEC using UVA, DOC, and fluorescence detection. Water Res. 37, 4295-4303.

Her, N., Amy, G., Foss, D., Cho, J., Yoon, Y., Kosenka, P., 2002a. Optimization of method for detecting and characterizing NOM by HPLC-size exclusion chromatography with UV and on-line DOC detection. Environ. Sci. Technol. $36,1069-1076$

Her, N., Amy, G., Foss, D., Cho, J.W., 2002b. Variations of molecular weight estimation by HP-size exclusion chromatography with UVA versus online DOC detection. Environ. Sci. Technol. 36, 3393-3399.
Her, N., Amy, G., Sohn, J., Gunten, U., 2008. UV absorbance ratio index with size exclusion chromatography (URI-SEC) as an NOM property indicator. J. Water Supply Res. Technol. 57, 35-44.

Huber, S.A., Frimmel, F.H., 1994. Direct gel chromatographic characterization and quantification of marine dissolved organic-carbon using high-sensitivity doc detection. Environ. Sci. Technol. 28, 1194-1197.

Huber, S.A., Balz, A., Abert, M., Pronk, W., 2011. Characterisation of aquatic humic and non-humic matter with size-exclusion chromatography-organic carbon detection-organic nitrogen detection (LC-OCD-OND). Water Res. 45, 879-885.

Hur, J., Kim, G., 2009. Comparison of the heterogeneity within bulk sediment humic substances from a stream and reservoir via selected operational descriptors. Chemosphere 75, 483-490.

Korshin, G.V., Li, C.W., Benjamin, M.M., 1997. Monitoring the properties of natural organic matter through UV spectroscopy: a consistent theory. Water Res. 31, 1787-1795.

Korshin, G., Chow, C.W.K., Fabris, R., Drikas, M., 2009. Absorbance spectroscopybased examination of effects of coagulation on the reactivity of fractions of natural organic matter with varying apparent molecular weights. Water Res. 43, 1541-1548.

Leenheer, J.A., Croue, J.P., 2003. Characterizing aquatic dissolved organic matter. Environ. Sci. Technol. 37, 18a-26a.

Li, C.W., Benjamin, M.M., Korshin, G.V., 2006. Characterization of NOM and its adsorption by iron oxide coated sand (IOCS) using UV and fluorescence spectroscopy. J. Environ. Eng. Sci. 5, 467-472.

Liu, S., Lim, M., Fabris, R., Chow, C., Drikas, M., Amal, R., 2010a. Comparison of photocatalytic degradation of natural organic matter in two Australian surface waters using multiple analytical techniques. Org. Geochem. 41, 124-129.

Liu, S., Lim, M., Fabris, R., Chow, C.W.K., Drikas, M., Korshin, G., Amal, R., 2010b. Multi-wavelength spectroscopic and chromatography study on the photocatalytic oxidation of natural organic matter. Water Res. 44, 2525-2532.

O'Loughlin, E., Chin, Y.P., 2001. Effect of detector wavelength on the determination of the molecular weight of humic substances by high-pressure size exclusion chromatography. Water Res. 35, 333-338.

Peuravouri, J., Pihlaja, K., 1997. Molecular size distribution and spectroscopic properties of aquatic humic substances. Anal. Chim. Acta 337, 133-149.

Rosenberger, S., Laabs, C., Lesjean, B., Gnirss, R., Amy, G., Jekel, M., Schrotter, J.C., 2006. Impact of colloidal and soluble organic material on membrane performance in membrane bioreactors for municipal wastewater treatment. Water Res. 40, 710-720.

Twardowski, M.S., Boss, E., Sullivan, J.M., Donaghay, P.L., 2004. Modeling the spectral shape of absorption by chromophoric dissolved organic matter. Mar. Chem. 89, 69-88.

Wong, S., Hanna, J.V., King, S., Carroll, T.J., Eldridge, R.J., Dixon, D.R., Bolto, B.A. Hesse, S., Abbt-Braun, G., Frimmel, F.H., 2002. Fractionation of natural organic matter in drinking water and characterization by C-13 cross-polarization magic-angle spinning NMR spectroscopy and size exclusion chromatography. Environ. Sci. Technol. 36, 3497-3503.

Yan, M.Q., Wang, D.S., You, S.J., Qu, J.H., Tang, H.X., 2006. Enhanced coagulation in a typical North-China water treatment plant. Water Res. 40, 3621-3627.

Yan, M.Q., Wang, D.S., Shi, B.Y., Wei, Q.S., Qu, H.H., Tang, H.X., 2007. Transformations of particles, metal elements and natural organic matter in different water treatment processes. J. Environ. Sci. - China 19, 271-277. 